\title{
CRISPR-Cas, a novel tool for unraveling the mechanisms of eye-related diseases
}

Kawalpreet K Aneja

*Correspondence: kaneja@hotmail.com

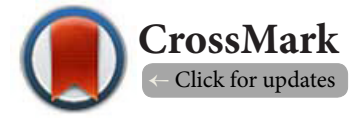

Research fellow, University of Pennsylvania, Philadelphia, PA 19104, USA.

\begin{abstract}
CRISPR-Cas is a novel tool that may pinpoint the underlying cause of eye diseases. Here, we introduce the different types of CRISPR-Cas systems with an emphasis on type II and present its applications in eye diseases based on previous reports. The CRISPR microbiome is perhaps bigger than the universe. Bacteria contain a self-defense adaptive immune system known as CRISPR (clustered regularly interspaced short palindromic repeats)-Cas (CRISPR-associated genes) which inactivates invading phages, plasmids, or foreign DNA sequences. CRISPR is an array of repeats separated by spacers along with cas genes. In bacteria CRISPR-array and cas genes together form a cascade complex to destroy the invading foreign DNA. The two important components, the Cas9 nuclease, and the short guide RNA are introduced for a targeted genetic event. The short guide-RNA is the fingerprint of the target DNA. CRISPR-Cas has been successfully demonstrated in RHO, CRYAA, tyr, gol, ddx19, and mitfa genes in zebrafish, mice, and rabbits. The nuclear homeoprotein Six3 mutations by CRISPR-Cas showed eye and anterior head defects in zebrafish. Moreover, CRISPR-Cas has been successfully shown in Crygc gene repair in the cataract mice model carrying 1bp deletion in exon3 of Crygc. The transcription factors like Rx, Six3, Pax6, Sox2, and signaling factors involved in eye formation can be further studied by CRISPR-Cas. The first patient ever to receive CRISPR-Cas9 construct for fixing mutations in a centrosomal protein of $290 \mathrm{kDa}$, CEP290, is for the blindness caused by a rare disorder in the retina, Leber's congenital amaurosis 10 (LCA 10). It is injected directly into the eye near photoreceptor cells and is a landmark clinical trial. The present CRISPRCas technology opens a new perspective for new treatments of cataract, retinitis pigmentosa, refractive index error, and any other eye diseases.
\end{abstract}

Keywords: CRISPR-Cas, CRISPR-array, Cas9 endonuclease, crRNA, sgRNA, Protospacer, PAM

\section{Introduction}

Bacterial cells have evolved two well-characterized systems to protect themselves against foreign DNA. One is the restrictionmodification system and the other is the CRISPR-Cas system. Both systems maintain and promote the survival of the bacterial genome. Restriction endonucleases perhaps provide protospacers for CRISPR-array. CRISPR repeats were first identified in E. coli $\mathrm{K} 12$ as identical 29-nucleotide repeat sequences separated by 32-nucleotide nonrepetitive sequences [1]. The CRISPR operon is made up of CRISPR array of repeats and spacers along with cas genes encoding CRISPR-associated proteins.

CRISPR-arrays have been found in both bacteria and archaea. Their prevalence was evident once the microbial genomic sequences were available, particularly E. coli strains. The entire system, however, was characterized only after the discovery of a link between CRISPR-array and its role in protection against foreign DNA (2). There are three main steps of CRISPR-Cas system (Figure 1): 1) Acquisition/Adaptation: foreign DNA fragment is cleaved and become part of a spacer at or near the leader sequence 2) Expression: CRISPR-array containing foreign DNA is processed into mature CRISPR RNAs (crRNAs) 3) Interference: Cleavage of foreign or invader DNA guided by crRNAs [2-4].

Currently, more than 7000 archaea and bacterial genomes have been found to contain one or more kinds of CRISPR-Cas systems [5]. In total, 11 CRISPR-Cas systems have been identified so far and have been classified into Type I, II, III, and their respective subtypes [4-7]. One interesting phenomenon is the co-occurrence of more than one CRISPR-Cas system in some 
bacteria. For example, Tistrella mobilis, and Azospirillum B510 contain III-A, I-C, and II-C [7]. Streptococcus thermophilus has four different types of CRISPR-Cas systems: III-B, I-E, II-A, and II-B [6-8].

Type II CRISPR-Cas system is the most widely used system for gene editing, gene repair, gene regulation, and gene visualization experiments. It can be rapidly used for single or multiple gene function analysis and it has been successfully used for making deletions of up to $1 \mathrm{Mb}$ [9]. Our review is an attempt to discuss this phenomenal bacterial defense system in the understanding and treatment of eye disorders.
We have discussed the known types of CRISPR systems with emphasis on type II. We are also presenting the experimental data employing CRISPR-Cas in the study of different eye genes and discuss the future of this technology.

\section{Review}

\section{Type I CRISPR-Cas System}

There are three known types of CRISPR-Cas systems: Type I, II, and III. Each is made up of a CRISPR array and cas genes associated with the array (Table 1). Type I is classified into six subtypes from I-A to I-F. Type I-E has been studied in E. coli.

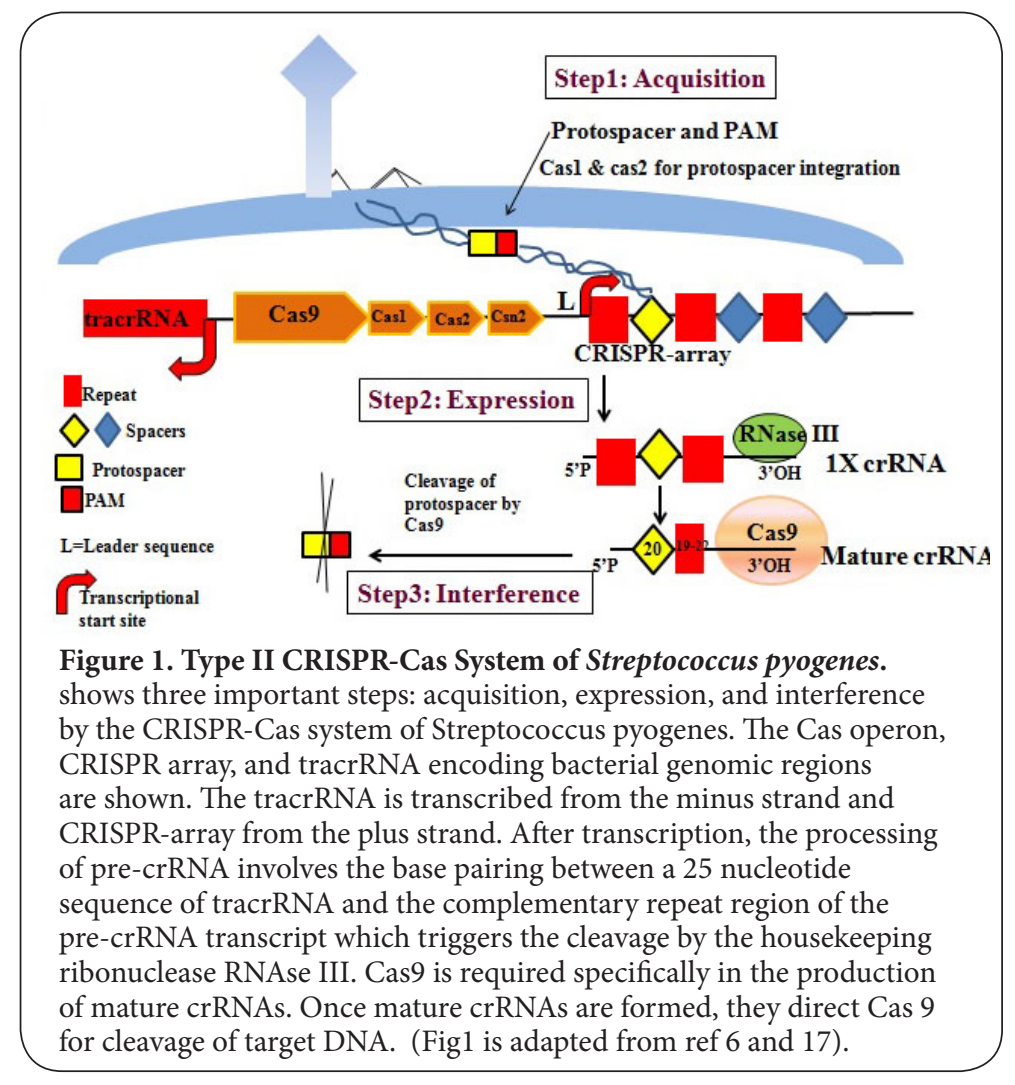

Table 1. Major CRISPR-Cas systems, their signature genes, and PAM sequence.

\begin{tabular}{|c|c|c|c|c|c|c|}
\hline System & Subtypes & Signature genes & Acquisition & Expression & Interference & PAM \\
\hline Type I & Six I-A to I-F & $\begin{array}{l}\text { Cas1, Cas2, Cas3, } \\
\text { Cas6 }\end{array}$ & Cas1, Cas2 & Cas6 & Cas3 & $\begin{array}{l}\text { 5'-CTT-3' } \\
\text { (E. coli) }\end{array}$ \\
\hline Type II & $\mathrm{A}, \mathrm{B}$, and $\mathrm{C}$ & $\begin{array}{l}\text { Cas1, Cas2, Csn2, } \\
\text { Cas9 }\end{array}$ & Cas1, Cas2 & $\begin{array}{l}\text { RNase III, } \\
\text { tracrRNA } \\
\text { Cas9 }\end{array}$ & Cas9 & $\begin{array}{l}\text { 5'-NGG-3' } \\
\text { (S. pyogenes) } \\
\text { 5'-NNAGAAw-3' } \\
\text { (S. thermophilus) } \\
\text { 5'-NGGNG-3' } \\
\text { (S. thermophilus) }\end{array}$ \\
\hline Type III & $\mathrm{A}$ and $\mathrm{B}$ & $\begin{array}{l}\text { Cas1, Cas2, Csm or } \\
\text { Cmr complex, Cas6, } \\
\text { Cas10 }\end{array}$ & Cas1, Cas2 & $\begin{array}{l}\text { Cas6, Csm or } \\
\text { Cmrcomplex }\end{array}$ & Not clear & No PAM \\
\hline
\end{tabular}

Adapted from Staals et al., 2013 [3], Makarova et al., 2011 [4], and Carte et al., 2014 [6], Semenova et al., 2011 [10], Wiedenheft et al., 2011 [11], Mojica et al., 2009 [13], Jinek et al., 2012 [14], Brouns et al., 2008 [62], Jore et al., 2011 [63], Westra et al., 2012 [64], Garneau et al., 2010 [65], Sapranauskas et al., 2011 [66]. 
Type I-E locus consists of 8 genes: cas 3 , cse 1, cse2, cas7, cas5, cas6e, cas 1, and cas2 [3]. These cas genes are located upstream of the CRISPR array. CRISPR arrays are typically made up of 29 nucleotide palindromic repeats that are separated from each other by 32-33 nucleotide spacer sequences [3]. Some of these repeats make secondary structures which are important for CRISPR-RNA (crRNA) maturation. Cas6e is a signature gene, a metal-independent endoribonuclease; it cleaves the repeat sequence at the conserved position, 8-nucleotides upstream of the repeat-spacer boundary, and helps in the formation of mature crRNAs. Once the mature crRNA is made, Cas6e guides the cascade complex to complementary target DNA $[3,4]$. Another signature gene Cas 3 is a nuclease/helicase and is required for the target degradation along with Cas6e [3] (Table 1). In Type I-E system PAM (protospacer adjacent motif) sequence CTT is the key for recognition [3]. PAM sequence is present in the foreign DNA (protospacer). PAM and a minimum of seven crRNA-complementing nucleotides in the protospacer (the so-called 'seed sequence') allow for CRISPR interference to take place $[3,10,11]$.

\section{Type III CRISPR-Cas System}

Type III CRISPR-Cas systems are present in $80 \%$ of archaea and $40 \%$ of bacteria [12]. It has been identified in bacterial pathogens such as Staphylococci and Mycobacteria and a clinical Staphylococcus epidermidis isolate. The signature genes of type III are Cas6 and Cas 10 (Table 1). The other hallmark of type III is RAMPs; multiple genes that encode "repeat-associated mysterious proteins or RAMPs" [12]. RAMPS are a superfamily of Cas proteins part of Csm or Cmr complex, possessing one or more RNA recognition motifs and a characteristic glycine-rich loop $[4,12]$. Cas6, Cas 10, and RAMPS help in processing and maturation of crRNAs and play roles in the defense phase of CRISPR interference [12]. PAM sequence is not required for targeting in type-III systems $[12,13]$. There is a beautiful example of how bacteria prevent auto-immunity while protecting their genome from foreign invasion. The $5^{\prime}$-tag on the crRNAs of Staphylococcus epidermidis is essential for targeting and most likely constitutes a seed sequence [12]. The flanking repeat sequence upstream of $5^{\prime}$ region licenses the foreign DNA for interference, how; the perfect complementarity between these two regions prevents targeting, the mismatches result in targeting foreign nucleic acid $[4,12]$. That is how $S$. epidermidis distinguishes self from non-self [12].

\section{Type II CRISPR-Cas System}

Type II is the most widely used system for genome editing. It is the rarest of all CRISPR-Cas systems missing in archaea and present in 5\% of the bacterial genomes [4-7]. Type II has three subtypes based upon their characteristic operon organization: II-A, II-B, and II-C. Type IIA contains an additional gene, csn2, which is involved in spacer integration, for example in S. pyogenes SF370 [58]. Csn2 protein exists in both long and short forms $[7,8]$. Type IIB has cas 4 instead of csn2 and it strictly maintains the spacer selection with the right PAM sequence, for example, Synechocystis sp. 6803 [7,8]. Type IIC has been identified in Neisseria meningitides. It is different from the other type II forms because crRNAs in this type are transcribed from promoters that are embedded within each CRISPR repeat, so crRNA 5' ends are transcribed [7] and crRNA 3' end formation requires processing by RNAase III and tracrRNA [60].

Type-II system minimally contains CRISPR-array and 3-4 cas genes [7]. The ubiquitous cas 1 and cas 2 genes are required for spacer acquisition and the signature cas 9 gene (Table 1) has multiple roles. The cas 9 gene is the main endonuclease which recognizes and cleaves target DNA [7,10-17]. Cas9 is a large protein with two distinct domains: $\mathrm{MsrA} / \mathrm{HNH}$ and RuvC/RNaseH [17]. The HNH domain cleaves DNA strand that is complementary to the guide RNA sequence (target strand) and RuvC/RNase $\mathrm{H}$ cleaves DNA strand that is opposite to the complementary strand (non-target strand). Both $\mathrm{HNH}$ and RNase $\mathrm{H}$ of Cas9 act as a molecular anchor to permit the pairing of tracrRNA with pre-crRNA $[6,14]$ and finally cleave the foreign DNA.

Type II is different from type I and type III because it uses a specialized RNA molecule known as trans-activating crRNA (tracrRNA) and RNase III instead of Cas-endoribonuclease (in type I and III, mainly Cas6). The tracrRNA sequence is located either between cas 9 and cas 1 or upstream of the cas 9 gene $[6,17,18]$. The tracrRNA orthologs are characterized by the presence of an antirepeat sequence homologous to cognate CRISPR repeats $[7,8]$. The first processing event involves base-pairing of tracrRNA with pre-crRNA repeats in the presence of Cas9 to form RNA duplexes that are cleaved by the endogenous RNase III. Cas9 is an anchor for the base-pairing of tracrRNA with pre-crRNAs $[17,18]$. The intermediate crRNA species or crRNA1X [6] undergoes a further maturation event resulting in mature individual smaller units called crRNAs that remain duplexed with tracrRNA in a complex with the Cas9 protein [11]. Cas9 is only required during the second cleavage step from crRNA1X to mature crRNAs (Figure 1) [6]. The tracrRNA also undergoes cleavage in parallel with crRNAs [6]. In S. pyogenes, the final crRNA molecule is made up of a single spacer containing $\sim 1 / 3$ of guide sequence and a partial CRISPR repeat of 19-22 nucleotides [12] (Figure 1). The way pre-crRNA is processed from long precursor to final mature CRISPR-RNA varies among different species.

A PAM sequence is present near the protospacer region. PAM sequences in CRISPR-Cas of $S$. thermophilus are 5'-NNAGAAW-3' (W for A/T) and 5'-NGGNG-3 while in S. pyogenes is $5^{\prime}$-NGG-3' (Table 1) [8]. PAM sequence helps in recognizing the foreign protospacer region. The PAM-interacting domain of Cas9 guides Cas1-Cas2 complex in selecting protospacers [61]. CRISPR protein complex cleaves the protospacer DNA and is then incorporated into the leader end of CRISPR array. During this spacer acquisition stage, a new repeat is also added by an unknown mechanism. Deletions of one or more old spacers have been also detected [8]. 
CRISPR-Cas system containing cells are primed to efficiently combat a foreign element that contains a matching protospacer and PAM. Cleavage occurs within the protospacer specifically 3 nucleotides upstream of the PAM sequence in most of the cases of S. thermophilus DGCC7710 and S. pyogenes [8]. In the interference stage, it is assumed that the crRNA and its associated Cas proteins are guided to the matching foreign dsDNA. The specific domains of Cas9 recognize this hybrid structure and cleave the target dsDNA.

CRISPR-spacers mainly appear from the phage genome, mobile genetic elements, and mysterious elements that comprise the 'dark matter' of CRISPR [61]. It was found that one-third of the spacer sequences had no obvious extrachromosomal origin $[19,20]$. Three CRISPR1 spacers and one CRISPR3 spacer showed $100 \%$ identity to $S$. thermophilus chromosomal sequences, notably in DtpT (a proton symporter), RexA (an ATPdependent exonuclease), and Ster_0775 (a phage-associated DNA primase and an intergenic region (between Ster_0810 and Ster_0811 genes) $[\mathbf{8}, \mathbf{1 4}]$. Thus, it is proposed that CRISPRCas systems may play a role in microbial genome regulation by controlling mRNA transcript levels $[8,15,19,20]$. The dark matter of the CRISPR array, the unknown spacers may also comprise the missing phage and mobile genetic elements sequences [61].
Applications of CRISPR Technology in Ophthalmology The discovery of Cas 9 endonuclease has resulted in a faster and less expensive way of genome editing or genomic surgery [20-23], but one must be careful for mismatches or off-targets. Cas9 endonuclease has two highly conserved domains: RuvC and $\mathrm{HNH}$ as mentioned before. The RuvC domain targets the non-complementary strand and the $\mathrm{HNH}$ domain cleaves the complementary target DNA strand [17,57]. Upon complementarity of guide RNA and target DNA, a heteroduplex is formed and then Cas9 cleaves the target DNA [23,57]. Sequences fully complementary to the guide RNA but lacking a nearby PAM are ignored by Cas $9[17,23,57]$. In the absence of recognition of PAM sequence the residency time of Cas 9 is very short $[17,57]$. The proximal 10-12 nucleotide near $3^{\prime}$ end of the spacer are the seed region, any mismatches in the seed region would prevent Cas9 from executing the cleavage of target, and any homologies in the seed region would cause promiscuity of Cas 9 [57]. Once PAM is bound it results in the unwinding of target DNA and efficient DNA cleavage $[17,57]$. The Cas9 along with dual guide RNA and tracrRNA is a great external artificial system for mutating genes in a variety of organisms from bacteria to humans. To make mutations, Cas9 nuclease and short guide RNA (containing both crRNA and tracrRNA) are introduced into the host of choice (Figures $\mathbf{2 a}$ and $\mathbf{2} \mathbf{b}$ ).

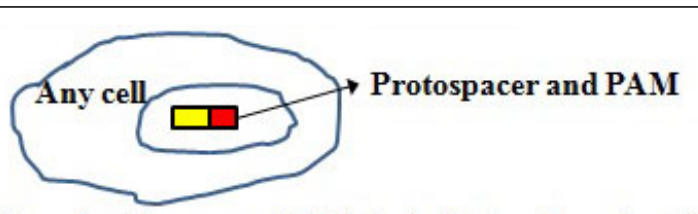

The target sequence of approximately 20-nucleotides next to PAM site is designed or selected by CRISPR software. Then it is cloned without PAM sequence into a plasmid containing the promoter of choice and tracrRNA scaffold. CRISPR Software websites: http://crispr.mit.edu/

(a) http://wwww.e-crisp.org/E-CRISP/designcrispr.html

Two important plasmids for CRISPR targeting.

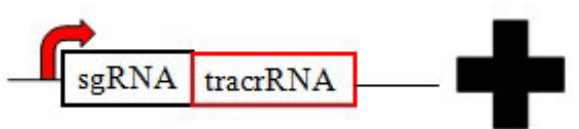

Short guide RNA (sgRNA) expression vector containing 20 nucleotides matching with the target DNA and fused with tracrRNA.

Transcriptional start site

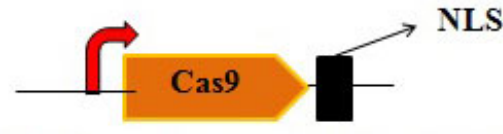

Cas 9 expression vector: Cas 9 nuclease is fused with nuclear localization signal (NLS).

Figure 2. CRISPR-Cas design for eye gene of interest.

Shows how to design CRISPR-Cas for gene targeting. Adapted from the Addgene website (https://www.addgene.org/CRISPR/guide/). The short guide RNA and Cas9 endonuclease can be on the same or two different plasmids. Cas9 applications: endonuclease, nickase, by inactivating Cas9 catalytic domain- Cas9 can be used as a fusion protein containing DNA binding domain of Cas9 + transcriptional activator or repressor, cytidine or thymidine deaminase, the fusion of histone modifiers for epigenetic regulation, the fusion of fluorescent proteins for imaging of genomic loci. 
Cas9 endonuclease makes a double-stranded break and can tolerate certain mismatches in the complementary sequence and results in off-target events [22-25]. Off-targets are highly undesirable, especially for therapeutic applications. The enzyme Cas9 nickase only makes nicks in single strands and nicks can be repaired with high fidelity. Mutated Cas9 $\mathrm{HNH}$ (H840A) and RuvC (D10A) have been engineered as a Cas9 nickase (dCas9); they result in a single-stranded DNA break $[24,25]$, and use the same base-excision repair pathway, but with little or no damage to the genome or off-targets. So, dCas9 is used with two guide RNAs separated by no more than 10 bps to make nicks on the opposite strands.

Experiments have demonstrated that dCas 9 can be used for gene activation or repression studies $[\mathbf{2 6 , 2 7 ]}$. The dCas9 fused with activation domain VP64 along with multiple sgRNAs showed enhanced expression of ASCL1, NANOG, HBG1and HBG2, MyoD1, VEGFA, TERT, IL1B, and IL1R1 promoters in HEK293T cells [26]. Similarly, it has been used for targeted repression by fusing dCas 9 with transcriptional repression domain (the Krüppel-associated box (KRAB) domain) [27].

CRISPR-Cas mediated dsDNA breaks can be repaired by either nonhomologous end-joining (NHEJ) or homologydirected repair (HDR) pathways. These pathways exist in all types of cells and organisms [28]. HDR pathway can use another homologous strand which is nearby, for example, in S-phase or it can be delivered exogenously in the form of oligonucleotide fragment or a plasmid containing homologous regions [29]. During repair by NHEJ pathway, there is an efficient introduction of insertion/deletion mutations (indels) of various lengths [29]. But in HDR, we can insert the desired point mutation or sequence at dsDNA break. Indels are possible even with HDR. The Mutation rate by any of these methods is typically greater than $1 \%$ and in some cases over $50 \%$ [29].

Correction and Modeling of Cataract-Inducing Mutations A cataract is a clouding of the lens and for a patient with cataract; vision is like seeing through a frosty or fogged-up window. A cataract is mainly an age-related disease but congenital cataracts are a leading cause of blindness in newborns. Congenital cataracts are mainly caused by autosomal dominant or recessive mutations in the $\alpha \mathrm{A}$-crystallin gene (CRYAA) and $\alpha \mathrm{B}$-crystallin (CRYAB) gene. Congenital cataract has been modeled in rabbits by knocking out $\alpha A$-crystallin gene. The rabbit zygotes were co-injected with Cas9mRNA and sgRNA against $\alpha A$-crystallin gene. These rabbits showed phenotypes of congenital cataracts, microphthalmia, obscurity, early atrophy of the lens, and failed differentiation of lens fibers and these rabbits showed a lack of $\alpha A$-crystallin protein expression [29]. This new CRISPR-Cas created cataract rabbit model is not only useful for studying the role of crystallin in human cataract but can also help in conducting drug screening for cataract prevention in clinical practice. Recently, the CRISPR-Cas system has been used to reverse the cataract phenotype of mice carrying a dominant mutation in the
Crygc gene [30]. These mice carry a $1 \mathrm{bp}$ deletion in exon 3 of the Crygc gene which results in a shifted reading frame and introduction of a stop codon at the $76^{\text {th }}$ amino acid of exon 3. The consequence of this deletion is the production of a truncated, dominant-negative $\gamma \mathrm{C}$-crystallin and ultimately, the formation of cataracts $[30,31]$. To repair this mutation, the mammalian codon-optimized Cas9 mRNA and single-guide RNA (sgRNA) with or without single-stranded oligonucleotide donor were injected into B6D2F1 mouse zygotes carrying one allele of mutant Crygc. When Cas9 mRNA and sgRNA were injected without oligo-donor, out of 135 transferred blastocysts, a total of 22 live pups were born and 4 were corrected of cataracts with HDR-mediated repair by internal wild allele and 2 by NHEJ-mediated indels. By injecting oligo-donor 1,9 out of 29 live pups were free of cataracts and 5 pups showed correction by HDR-directed repair. To further investigate the efficiency of HDR-repair by external oligo-donor, another oligo-donor carrying two synonymous mutations were used. In this case, 9 out of 27 were free of cataracts and 4 were cured via HDR; three of them carried modified Crygc genes with a DNA sequence that was the same as the exogenous oligo-donor, and remaining one had a DNA sequence identical to that of the WT allele [30]. In this experiment mutation repair by using NHEJ or HDR did not show a big difference. The exogenous oligonucleotide can increase the efficiency of homology-directed repair by acting as a template for repairing Cas9-mediated double-strand breaks and replacement of mutant alleles and is especially useful when endogenous wild type allele cannot be used as the template $[30,31]$. Out of 12 of 22 mice, 2 showed off-target mutations at one of the potential off-target sites. In this study, however, the frequency of correction or repair was low and this may be improved by better optimized Cas9 endonuclease, or Cas9 nickase, better sgRNA or improved injection methods.

A similar CRISPR-Cas-mediated correction has been done in the Fah5981SB mouse model of human hereditary tyrosinemia (HTI). The Fah5981SB mouse is homozygous for a $G$ to $A$ point mutation of the last nucleotide of exon 8 in the fumarylacetoacetase (FAH) gene results in truncated, unstable FAH protein, which mimics human HTI. FAH deficiency causes accumulation of toxic metabolites, such as fumarylacetoacetate, in hepatocytes, resulting in severe liver damage. The plasmid pX330 coexpressing Cas9 and one sgRNA along with a 199 nucleotide, single-stranded DNA donor were given to adult Fah ${ }^{\text {mut/mut }}$ mice by hydrodynamic tail vein injections [32]. This resulted in wild-type FAH protein in $\sim 1 / 250$ liver cells [32]. This was sufficient to rescue the body weight loss phenotype, as improvement of liver histology and reduction of serum markers for liver health like aspartate aminotransferase, alanine aminotransferase, and bilirubin [32]. The above type of experiment was also performed in human induced pluripotent stem cells (iPSCs) using a combination of zinc finger nucleases (ZFNs) and piggyBac9 transposon. The piggyBac9 transposon is used for precise and scarless modification of the genome 
[33]. This experiment showed biallelic correction of a point mutation (Glu342Lys) in the a-antitrypsin (A1AT, also known as SERPINA1) gene that is responsible for a-antitrypsin deficiency [33]. We can use the same techniques for eye-disease related mutations.

Zebrafish is an excellent model for human congenital cataract. For example, a c.385 G>T variation in exon 3 of Crygc discovered in a Chinese family changes glycine to cysteine at codon 129 (p.Gly129Cys) and results in an autosomal dominant nuclear-cataract causing mutant protein. Zebrafish embryos injected with mutant P.Gly129Cys mRNA show increased vacuole formation in the lens, incomplete denucleation, and eye lens with the central cloudy region [34]. In this manner, the human cataract phenotype was just recapitulated in zebrafish embryos. Mutations in the PITX3 and FOXE3 genes, which are expressed in the lens epithelium, cause anterior segment defects, including cataracts, have been also modeled in zebrafish [35-37]. The availability of zebrafish genome sequence information and mutants from Sanger Institute makes modeling multiple cataract-inducing mutations in zebrafish possible. Experiments similar to those on Crygc can likewise be performed on a wide variety of heretofore, in the context of eye diseases, unexamined crystalline genes, connexins, and transcription factors such as FokC1, Pax6, Six 3 , PITX3, and HSF4.

\section{Elucidation of Eye Formation Pathways}

Some interesting experiments are done by ectopic expression of an eye on antennae or legs. The mouse transcription factor Pax6 (paired box 6) can induce the formation of the functional ommatidial eyes in Drosophila antennae or legs [38-40]. This shows that Pax 6 may be a switch for eye development. The expression of mouse Six 3 in the ear placode of the medaka fish led to the development of a lens [41]. Similarly, the injection of Six3mRNA into medaka embryos resulted in ectopic Pax6 expression and the formation of ectopic retinal primordia [41]. Human mutations in Six 3 lead to holoprosencephaly; in some cases, the phenotype is milder and manifests a microphthalmia and iris coloboma [41-45]. In humans, Pax6 mutations mainly cause aniridia, isolated cataracts, and Peters anomaly. The significance of Pax 6 has been further demonstrated through the use of injected TALENS (Transcription activator-like effector nucleases) against Pax6 which resulted in deformed eyes in Xenopus laevis embryos [45]. CRISPR-Cas induced mutations in the six 3 coding regions as well as in its promoter region showed anterior head and eye defects [46]. The experiments on Pax6 and Six 3 suggest that they play critical roles in early eye development. Six 3 is also activated byPax 6 and Prox 1 [43]. Prox 1 is another transcription factor important for the differentiation of lens fiber cells and a-crystallin expression [44]. $R X$ and SOX2 are retinal and anterior neural fold homeobox genes, critical for eye development [44]. RX-deficient mouse embryos lack eye anlagen and Pax6 expression in the eye field [44]. Therefore, $R x$, Six3, Pax6, and Sox 2 are involved in early eye development.

Defects in signaling molecules such as sonic hedgehog $(\mathrm{SHH})$ also appear to play important roles in eye development [42]. This can be seen in $\mathrm{SHH}$ knock-out mice and humans with $\mathrm{SHH}$ mutations which both show holoprosencephaly with ocular manifestations that range from microphthalmia to cyclopia [42]. The exact contributions of $\mathrm{SHH}$ and other signaling molecules such as BMP4 and BMP7 to eye development are not known. These molecules may regulate for early eye development transcription factors like $R X$, SiX3, Pax6, or other specific eye region transcription factors. Gene editing tools such as CRISPR-Cas can be used to elucidate these pathways. By mutating genes using Cas 9 endonuclease or nickase and comparison of phenotypes, additional important genes can be identified. CRISPR-Cas can also be used for visualization of endogenous genomic loci, repetitive, and non-repetitive genomic sequences [47]. Endonuclease deficient Cas9 fused with GFP can be directed by specifically designed sgRNAs for dynamic imaging. This system helped in robust imaging of repetitive elements in telomeres and coding genes in living cells [47]. We can similarly utilize this technique for imaging human eye coding genes. A new technique with inactive Cas9 fused to Fokl nuclease has been shown to enhance target specificity and less off-target effects [48]. All these new types of Cas 9 fusion proteins will provide more flexibility and understanding of the eye development pathways.

Use of Gene Editing in the Study of Other Eye Disorders Retinitis pigmentosa (RP) is caused by the progressive degeneration of the retina and retinal pigmented epithelium. It is characterized by the progressive peripheral and night vision loss, which depending on the phenotypic subtype, results in a fast or slow loss of central vision [49]. About $25 \%$ of autosomal dominant RP cases are connected to over 100 point mutations in the rhodopsin gene [49]. Identification of different mutations in the rhodopsin gene suggest that mistrafficking and misfolding of rhodopsin may be the cause of severe changes in vision loss though the mechanism behind these mutations is not understood. The Pro23His rhodopsin ( $\left.\mathrm{Rho}^{\mathrm{P} 23 \mathrm{H}}\right)$ mutation is commonly associated with autosomal dominant RP in North America and a stable human embryonic retinoblast cell line containing Pro23His rhodopsin has been created [50]. Zinc finger nucleases (ZFNS) against human rhodopsin gene in human embryonic retinoblasts showed a 12 -fold increase in homologous recombination and an absolute frequency of ZFN-directed homologous recombination as high as $17 \%$ in human rhodopsin gene [50]. But CRISPR-Cas mediated ablate-and-replace gene therapy showed promising results by rescuing the Rho ${ }^{\mathrm{P} 23 \mathrm{H}}$ mutation defect. By injecting codonoptimized Cas9, double gRNA to make a 300 bp deletion containing Rho ${ }^{\mathrm{P} 23 \mathrm{H}}$ mutation, and $\mathrm{hRHO}$ cDNA to replace the wild allele, driven by mouse $\mathrm{RHO}$ promoter. The $\mathrm{Rho}^{\mathrm{P} 23 \mathrm{H}}$ mice showed remarkable recovery from autosomal dominant RP. Histological and electroretinographical analysis showed $17 \%$ 
to $36 \%$ more outer nuclear layer thickness at three months after AAV injection; the $a$ and $b$ waves were preserved more significantly than just using gene replacement monotherapy [51]. The same strategy also showed promising results in Rho ${ }^{\mathrm{D} 190 \mathrm{~N}}$ mutation. This is a promising future therapy for human RP. Just like glial cells, Muller glial cells (MG) are adult retinal stem cells that have been implicated in retinal regeneration [52]. Muller glial cells are an injury-induced stem cell model in zebrafish. Zebrafish have a robust capacity for retinal repair and can regenerate whole retinas, somal layers, and even discrete retinal sub-neurons with help of highly regenerative MG cells. In humans, MG cells cannot regenerate, but cultured human MG cells can produce neurons capable of restoring visual function when transplanted into retinal degeneration models, hence they have the potential to act as stem cells and hold the strong therapeutic capacity for retinal regeneration. Using multiplexed CRISPR-Cas9 targeting of genes, 614 sites in 312 regeneration genes, and 14 sites in 8 RP-linked genes were screened by Automated Reporter Quantification assay. This led to the isolation of a novel mutation in rhodopsin $\left(\right.$ rho $\left.^{\mathrm{djh} h 03}\right)$, which encodes the G-protein coupled receptor necessary for phototransduction in rod cells [52].

CRISPR-Cas was used for mutations in tyrosinase (tyr), golden (gol), mitfa, and $d d x 19$ genes [53] in zebrafish. The tyr gene mutation showed mosaic pigmentation, patchy loss of pigmentation in the retinal pigment epithelium, golden gene showed hypopigmentation in RPE, mitfa involved in neural crest development did not show detectable phenotype and $d d x 19$ showed brain necrosis, small eyes, and curved body axis. Biallelic mutations by using TALENS in the tyrosinase (tyr) gene also showed oculocutaneous albinism in Xenopus tropicalis [54].

\section{Conclusion and Perspectives}

CRISPR-Cas has been successfully utilized in the study of pigmentation genes like tyrosinase (tyr) and golden ( $g o l)$. It has been used for mutations in ddx-19, mitfa, Pax6, gata4, and gata5 [55]. ZFNs have shown successful homology-directed repair of Pro23His mutation in the rhodopsin gene. CRISPR-Cas was applied to repair $1 \mathrm{bp}$ mutation of crygc gene in the mouse cataract model. Many genes, transcription factors, and signaling molecules are common to the brain and eye development. For example, Walker-Warburg syndrome (WWS) and muscle eye brain disorder (MEB) caused by glycosyltransferases which result in defective glycosylation. They both show common brain and eye abnormalities along with muscular dystrophy [56]. A new marker fukutin related protein (FKRP) with novel mutations has been discovered in two new patients of MEB and WWS [56]. We can visualize these common pathways or targets by exploiting inactive Cas 9 fused with fluorescence reporter GFP. The new form of inactive Cas9 fused with Fok1 endonucleases may enhance gene specificity and less off-target effects. CRISPR-Cas can be used to rapidly assess single or multiple gene functions. Large inversions/deletions from several $100 \mathrm{bps}$ to $1 \mathrm{Mb}(981 \mathrm{~kb})$ have been achieved in coding regions as well as in microRNA genes of zebrafish [55]. More efficient CRISPR-Cas systems with better gene targeting ability will soon offer new treatments for eye diseases. For example, CRISPR-from Streptococcus thermophilus, Neisseria meningitidis and Treponema denticola have been characterized. Hopefully, new systems will allow better recognition, fewer PAM sequence restrictions, and reduced off-target activity. In the future, more types of cas 9 endonucleases, nickases, and Fok1 nucleases will revolutionize mutant gene repair in a variety of eye disorders.

\section{List of Abbreviations}

Protospacer: Short fragment of foreign nucleic acid

PAM: Protospacer adjacent motif

CRISPR-array: DNA sequence of repeats and spacers

Cas9: CRISPR-associated protein 9

CrRNA: CRISPR RNA

sgRNA: short guide RNA

tracrRNA: trans-activating crRNA

Indels: Insertions/deletions

NHEJ: Nonhomologus end joining

HDR: Homology-directed repair

\section{Competing interests}

The author declares that she has no competing interests.

Acknowledgements

I am extremely thankful to Dr. Dwight Stambolian and Dr. Harvey Rubin for giving me inspiration to write this article.

Publication history

Editor: Carsten H. Meyer, Pallas Clinic, Switzerland.

Received: 27-July-2020 Final Revised: 30-Aug-2020

Accepted: 10-Sept-2020 Published: 22-Sept-2020

\section{References}

1. Ishino Y, Shinagawa H, Makino K, Amemura M, Nakata A. Nucleotide sequence of the iap gene, responsible for alkaline phosphatase isozyme conversion in Escherichia coli, and identification of the gene product. J. Bacteriol 1987; 169:5429-5433 PubMed

2. Mojica, FJ, and Garrett, RA. Discovery and seminal development in the CRISPR field. In CRISPR-Cas Systems (Barrangou, R. and van der Oost, J., eds), 2013; pp. 1-26, Springer-Verlag, Berlin

3. Staals, RH and Brouns, SJ. Distribution and mechanism of the type I CRISPR-Cas system. In CRISPR-Cas Systems (Barrangou, R. and van der Oost, J., eds),2013; pp. 145-169, Springer-Verlag, Berlin

4. Makarova KS, Haft DH, Barrangou R, Brouns SJ, Charpentier E, Horvath P, Moineau S, Mojica FJ, Wolf YI, Yakunin AF, van der Oost J, eds and Koonin EV. Evolution and classification of the CRISPR-cas systems. Nature Reviews Microbiology 2011; 19: 467 PubMed

5. Grissa, I., Vergnaud, G., \& Pourcel, C. (2007). CRISPRFinder: a web tool to identify clustered regularly interspaced short palindromic repeats. Nucleic acids research 2007; 35(Web Server issue), W52-W57.

6. Carte J, Christopher RT, Smith JT, Olson S, Barrangou R, Moineau S, Glover CV, Graveley BR, Terns RM, Terns MP (2014) The three major types of CRISPR-Cas systems function independently in CRISPR RNA biogenesis in Streptococcus thermophilus. Molecular Microbiology 93: 98-112 PubMed

7. Chylinski K, Makarova KS. Charpentier E, and Koonin EV (2014) Classification and evolution of type II CRISPR-Cas systems. Nucleic Acids Research 42 (9): 1-15 PubMed

8. Dupuis ME and Moineau S (2013) Type II: Streptococcus thermophilus. 
Aneja, Journal of Eye and Ophthalmology 2020,

http://www.hoajonline.com/journals/pdf/2055-2408-7-1.pdf

doi: $10.7243 / 2055-2408-7-1$

(Barrangou, R. and van der Oost, J., eds), pp. 171, Springer-Verlag, Berlin PubMed

9. Xiao A, Wang Z, Hu Y, Wu Y, Luo Z, Yang Z, Zu Y, Li W, Huang P, Tong X, Zhu $Z$, Lin S, Zhang B (2013) Chromosomal deletions and inversions mediated by TALENs and CRISPR/Cas in zebrafish. Nucleic Acids Res 41: e141 PubMed

10. Semenova E, Jore MM, Datsenko KA, Semenova A, Westra ER, Wanner B, van der Oost J, Brouns SJ, Severinov K (2011) Interference by clustered regularly interspaced short palindromic repeat (CRISPR) RNA is governed by a seed sequence. Proc Natl Acad Sci USA 108(25): 10098-100103 PubMed

11. Mojica B, Lander GC, Zhou K, Jore MM, Brouns SJ, van der Oost J, Doudna, JA, Nogales E. (2011) Structures of the RNA-guided surveillance complex from a bacterial system. Nature 477(7365): 486-489 PubMed

12. Hatoum-Aslan A, Palmer KL, Gilmore MS, Marraffini LA (2013) Type III CRISPR-Cas systems and the roles of CRISPR-Cas in bacterial virulence. (Barrangou, R. and van der Oost, J., eds), pp. 201, Springer-Verlag, Berlin

13. Mojica FJ, Diex-Villasenor C, Garcia-Martinez J, Almendros C (2009) Short motif sequences determine the targets of the prokaryotic CRISPR defense system. Microbiology 155 (t3): 733-740 PubMed

14. Jinek M., Chylinski K, Fonfara I, Hauer M, Doudna JA, Charpentier E. (2012) A programmable dual RNA-guided DNA endonuclease in adaptive bacterial immunity. Science 337:816-821 PubMed

15. Horvath P, Dennis A. Romero, Anne-Claire Coûté-Monvoisin, Melissa Richards, Hélène Deveau, Sylvain Moineau, Patrick Boyaval, Christophe Fremaux, and Rodolphe Barrangou (2008) Diversity, Activity, and Evolution of CRISPR Loci in Steptococcus thermophilus. J Bacteriol 190(4): 1401-1412. PubMed

16. Martin J, Jiang F, Taylor, DW. Sternberg SH, Kaya E, Ma E, Anders C, Hauer M, Zhou K, Lin S, Matias K, lavarone AT, Emmanuelle Charpentier, Nogales E, Doudna JA (2014) Structures of Cas9 endonucleases reveal RNAmediated conformational activation. Science 343(6176): 1215 PubMed

17. Deltcheva E, Chylinski K, Sharma CM, Gonzales K, Chao Y, Pirzada ZA, Eckert MR, Vogel J, Charpentier E (2011) CRISPR RNA maturation by trans-encoded small RNA and host factor RNAse III. Nature 471(7340):602-607 PubMed

18. Bolotin A, Quinquis B, Sorokin A, Ehrlich SD (2005) Clustered regularly interspaced short palindrome repeats (CRISPRs) have spacers of extrachromosomal origin. Microbiology 151(8): 2551-2561 PubMed

19. Makarova K, Slesarev A, Wolf Y, Sorokin A, Mirkin B, Koonin E, Pavlov A, Pavlova N Karamychev V, Polouchine N, Shakhova V, Grigoriev I, Lou Y, Rohksar D, Lucas S, Huang K, Goodstein DM, Hawkins T, Plengvidhya V, Welker D, Hughes J, Goh Y, Benson A, Baldwin K, Lee JH, Díaz-Muñiz I, Dosti B, Smeianov V, Wechter W, Barabote R, Lorca G, Altermann E, Barrangou R, Ganesan B, Xie Y, Rawsthorne H, Tamir D, Parker C, Breidt F, Broadbent J, Hutkins R, O'Sullivan D, Steele J, Unlu G, Saier M, Klaenhammer T, Richardson P, Kozyavkin S, Weimer B, Mills D (2006) Comparative genomics of the lactic acid bacteria. Proc Natl Acad Sci USA 103(42):15611-15616 PubMed

20. Mali P, Yang L, Esvelt KM, Aach J, Guell M, DiCarlo JE, Norville JE, Church GM (2013) RNA-guided human genome engineering via Cas9. Science 339(6121):823-826 PubMed

21. Cong L, Ran FA, Cox D, Lin S, Barretto R, Habib N, Hsu PD, Wu X, Jiang W, Marraffini LA, Zhang F (2013) Multiplex genome engineering using CRISPR/Cas systems. Science 339: 819-823 PubMed

22. Hwang WY, Fu Y, Reyon D, Maeder ML, Tsai SQ, Sander JD, Peterson RT, Yeh J, Keith J (2013) Efficient genome editing in zebrafish using a CRISPR-Cas system. Nature Biotechnology 31: 227-229 PubMed

23. Sternberg SH, Redding S, Jinek M, Greene EC, Doudna JA (2014) DNA interrogation by the CRISPR RNA-guided endonuclease Cas9. Nature 507: 62-67 PubMed

24. Ran FA, Hsu PD, Lin C, Gootenberg JS, Konermann S, Trevino AE, Scott DA, Inoue A, Matoba S, Zhang Y, Zhang F (2013) Double Nicking by RNAguided CRISPRCas9 for enhanced genome editing specificity. Cell 154: 1380-1389 PubMed

25. Bin S, Wensheng Z, Jun Z, Jiankui Z, Jianying W, Chen L, Wang L, Hodgkins
A, lyer V, Huang X, Skarnes WC (2014) Efficient genome modification by CRISPR-Cas9 nickase with minimal off-target effects. Nature Methods 11: 399-402 PubMed

26. Perez-Pinera P, Kocak DD, Vockley CM, Adler AF, Kabadi AM, Polstein LR, Thakore PI, Glass KA, Ousterout DG, Leong KW, Guilak F, Crawford GE, Reddy TE, Gersbach CA. (2013) RNA-guided gene activation by CRISPRCas9-based transcription factors. Nat Methods 10: 973-976 PubMed

27. Gilbert LA, Larson MH, Morsut L, Liu Z, Brar GA, Torres SE, Stern-Ginossar N, Brandman O, Whitehead EH, Doudna JA, Lim WA, Weissman JS, Qi LS (2013) CRISPR-mediated modular RNA-guided regulation of transcription in eukaryotes. Cell 154 (2): 442-451 PubMed

28. Sander, JD, Joung, JK, (2014) CRISPR-Cas systems for editing, regulating and targeting genomes. Nature Biotechnology 32(4): 347-355 PubMed

29. Yuan L, Yao H, Xu Y, Chen M, Deng J, Song Y, Sui T, Wang Y, Huang Y, Li Z, Lai L. (2017) CRISPR/Cas9-Mediated Mutation of $\alpha$ A-Crystallin Gene Induces Congenital Cataracts in Rabbits. Invest Ophthalmol Vis Sci. 58(6) BIO34-BIO41

30. Wu Y, Liang D, Wang Y, Bai M, Tang W, Bao S, Yan Z, Li D, Li J (2013) Correction of a genetic disease in mouse via use of CRISPR-Cas9. Cell Stem Cell 13: 659-662 PubMed

31. Santana A, Waiswol M (2011) The genetic and molecular basis of congenital cataract. Arq Bras Oftalmol 74(2):136-42 PubMed

32. Yin H, Xue W, Chen S, Bogorad RL, Benedetti E, Grompe M, Koteliansky V, Sharp PA, Jacks T, Anderson DG (2014) Genome editing with Cas9 in adult mice corrects a disease mutation and phenotype. Nature Biotechnology 32(6):551-3 PubMed

33. Yusa K, Rashid ST, Marchand HS, Varela I, Liu PQ, Paschon DE, Miranda E, Ordonez A, Hannan N, Rouhani FJ, Darche S, Alexander G, Marciniak SJ, Fusaki N, Hasegawa M, Holmes MC, Di Santo JP, Lomas DA, Bradley A, Vallier $L$ (2011) Targeted gene correction of a1-antitrypsin deficiency in induced pluripotent stem cells. Nature 478: 391 PubMed

34. Li X, Cai H, Zhou S, Yang J, Xi Y, Gao X, Zhao W, Li P, Zhao G, Tong Y, Bao F, Ma Y, Wang S, Yan Y, Lu C, Ma Xu (2012) A novel mutation impairing the tertiary structure and stability of $\gamma C$-Crystallin (CRYGC) leads to cataract formation in humans and zebrafish lens. Human Mutation 33(2): 391-401 PubMed

35. Semina EV, Ferrell RE, Mintz-Hittner HA, Bitoun P, Alward WL, Reiter RS, Funkhauser C, Daack-Hirsch C, Murray JC (1998) A novel homeobox gene PITX3 is mutated in families with autosomal-dominant cataracts and ASMD. Nat Genet 19(2): 167-170 PubMed

36. Semina EV, Brownell I, Mintz-Hittner HA Murray JC, Jamrich M. (2001) Mutations in the human forkhead transcription factor FOXE3 associated with anterior segment ocular dysgenesis and cataracts. Human $\mathrm{Mol}$ Genet 10(3): 231-236 PubMed

37. Dutta S, Dietrich JE, Aspock G, Burdine RD, Schier A, Westerfield M, Varga ZM. (2005) pitx3 defines an equivalence domain for lens and anterior pituitary placode. Development 132(7): 1579-1590 PubMed

38. Halder G, Callaerts P, Gehring WJ (1995) Induction of ectopic eyes by targeted expression of the expression of the eyeless gene in Drosophila. Science 267, 1788 PubMed

39. Chow RL, Altmann CR, Lang RA, Hemmati-Brivanlou A. (1999) Pax6 induces ectopic eyes in a vertebrate. Development 126: 4213-4222 PubMed

40. Oliver G, Loosli F, Köster R, Wittbrodt J, Gruss P (1996) Ectopic lens induction in fish in response to the murine homeobox gene Six3. Mech Dev 60 (2): 233-239 PubMed

41. Loosli F, Winkler S, Wittbrodt J (1999) Six3 overexpression initiates the formation of ectopic retina. Genes Dev 13: 649-654 PubMed

42. Jochen Graw (2003) The genetic and molecular basis of congenital eye defects. Nat Rev Genet 14: 876 PubMed

43. Lengler J, Graw J (2001) Regulation of the human SIX3 promoter. BBRC 287: 372-376 PubMed

44. Goudreau G, Petrou P, Reneker LW, Graw J, Loster J, Gruss P (2002) Mutually regulated expression of Pax6 and Six 3 and its implications for the Pax6 haploinsufficient lens phenotype. Proc Natl Acad Sci USA 99: 8719-8724 PubMed

45. Suzuki KT, Isoyama Y, Kashiwagi K, Sakuma T, Ochiai H, Sakamoto N, 
Furuno N, Kashiwagi A, Yamamoto T(2013) High efficiency TALENs enable FO functional analysis by high targeted gene disruption in Xenopus laevis embryos. Biology Open 448-452 PubMed

46. Nakayama T, Fish MB, Fisher M, Oomen-Hajagos J, Thomsen GH, Grainger RM. (2013) Simple and efficient CRISPR/Cas9-mediated targeted mutagenesis in Xenopus tropicalis. Genesis 51(12): 835-43 PubMed

47. Chen B, Gilbert LA, Cimini BA, Schnitzbauer J, Zhang W, Li Gene-Wei, Park J, Blackburn EH, Weissman JS, Qi LS, Huang B (2013) Dynamic imaging of genomic loci in living human cells by an optimized CRISPR/Cas system. Cell 155: 1479-1491 PubMed

48. Tsai SQ, Wyvekens N, Khayter C, Foden JA, Thapar V, Reyon D, Goodwin MJ, Aryee MJ, Joung JK (2014) Dimeric CRISPR RNA-guided FokI nucleases for highly specific genome editing. Nat Biotechnol 1-9 PubMed

49. Rakoczy EP, Kiel C, McKeone R, Stricher F, Serrano L (2011) Analysis of disease-linked rhodopsin mutations based on structure, function, and protein stability calculations. Journal of Molecular Biology 405: 584-606 PubMed

50. Greenwald DL, Cashman SM, Singh RK (2010) Engineered zinc finger nuclease-mediated homologous recombination of the human rhodopsin gene. Invest Ophthalmol Vis Sci 51(12): 6374-6380 PubMed

51. Tsai, Yi-Ting et al. (2018) Clustered Regularly Interspaced Short Palindromic Repeats-Based Genome Surgery for the Treatment of Autosomal Dominant Retinitis Pigmentosa. Ophthalmology 125:14211430 PubMed

52. Unal Eroglu, A., Mulligan, T. S., Zhang, L., White, D. T., Sengupta, S., Nie, C., Mumm, J. S. (2018). Multiplexed CRISPR/Cas9 Targeting of Genes Implicated in Retinal Regeneration and Degeneration. Frontiers in cell and developmental biology, 6, 88.

53. Jao LE, Wente SR, Chen W (2013) Efficient multiple biallelic zebrafish genome editing using a CRISPR nuclease system. Proc Natl Acad Sci USA 110 (34): 13904-13909 PubMed

54. Blitz IL, Biesinger J, Xie X, Cho KW (2013) Biallelic genome modification in FO Xenopus tropicalis embryos using the CRISPR/Cas system. Genesis 51:827-834 PubMed

55. Chang N, Sun C, Gao L, Zhu D, Xu X, Zhu X, Xiong JW, Xi JJ (2013) Genome editing with RNA-guided Cas9 nuclease in zebrafish embryos. Cell Research 23:465-47 PubMed

56. Beltran-Valero de Bernabe D, Voit T, Longman C, Steinbrecher A, Straub V, Yuva Y, Herrmann R, Sperner J, Korenke C, Diesen C, Dobyns WB, Brunner HG, van Bokhoven H, Brockington M, Muntoni F (2004) Mutations in the FKRP gene can cause muscle-eye-brain disease and Walker-Warburg syndrome. J Med Genet 42: e61 PubMed

57. Jiang F. and Doudna JA. (2017) CRISPR-Cas9 structures and mechanisms. Ann. Rev. Biophys. 46: 505-529

58. Marraffini LA. The CRISPR-Cas system of Streptococcus pyogenes: function and applications. 2016 Apr 7. In: Ferretti JJ, Stevens DL, Fischetti VA, editors. Streptococcus pyogenes: Basic Biology to Clinical Manifestations [Internet]. Oklahoma City (OK): University of Oklahoma Health Sciences Center; 2016-

59. Ledford, H. (2020) CRISPR treatment inserted directly into the body for first time. Nature 579(7798):185 PubMed

60. Zhang Y, Heidrich N, Ampattu BJ, et al. Processing-independent CRISPR RNAs limit natural transformation in Neisseria meningitidis. Mol Cell. 2013; 50(4):488-503.

61. McGinn, J., Marraffini, L.A. (2019) Molecular mechanisms of CRISPR-Cas spacer acquisition. Nat Rev Microbiol 17, 7-12 PubMed

62. Brouns SJ, MM, Lundgren M, Westra ER, Slijkhuis RJ, Snijders AP, Dickman MJ, Makarova KS, Koonin EV, van der Oost J. (2008) Small CRISPR RNAs guide antiviral defense in prokaryotes. Science 321:960-964 PubMed

63. Jore $M M$, Lundgren $M$, van Duijn $E$, Bultema JB, Westra ER, Waghmare $S P$, Wiedenheft B, Pul U, Wurm R, Wagner R, Beijer MR, Barendregt A, Zhou K, Snijders AP, Dickman MJ, Doudna JA, Boekema EJ, Heck AJ, van der Oost J, Brouns SJ (2011) Structural basis for CRISPR RNA-guided DNA recognition by Cascade. Nat Struct Mol Biol 18:529-536 PubMed

64. Westra ER, van Erp PB, Kunne T, Wong SP, Staals RH, Seegers CL, Bollen
S, Jore MM, Semenova E, Severinov K, de Vos WM, Dame RT, de Vries $R$, Brouns SJ, van der Oost J (2012) CRISPR immunity relies on the consecutive binding and degradation of negatively supercoiled invader DNA by Cascade and Cas3. Mol Cell 46:595-605 PubMed

65. Garneau JE, Dupuis ME, Villion M, Romero DA, Barrangou R, Boyaval P, Fremaux C, Horvath P, Magadan AH, Moineau S (2010) The CRISPR/ Cas bacterial immune system cleaves bacteriophage and plasmid DNA. Nature 468:67-71 PubMed

66. Sapranauskas R, Gasiunas G, Fremaux C, Barrangou R, Horvath P, Siksnys V (2011) The Streptococcus thermophilus CRISPR/Cas system provides immunity in Escherichia coli. Nucleic Acids Res 39:9275-9282 PubMed

\section{Citation:}

Aneja KK. CRISPR-Cas, a novel tool for unraveling the mechanisms of eye-related diseases. J Eye Ophthalmol. 2020; 7:1. http://dx.doi.org/10.7243/2055-2408-7-1 